\title{
ATP measurement as an objective method to measure environmental contamination in 9 hospitals in the Dutch/Belgian border area.
}

\author{
Andreas van Arkel ( $\square$ a.vanarkel@etz.nl)
}

Elisabeth-TweeSteden Ziekenhuis https://orcid.org/0000-0003-3391-9200

Ina Willemsen

Amphia Ziekenhuis Locatie Molengracht

Linda Kilsdonk-Bode

Amphia Ziekenhuis Locatie Molengracht

Sindy Vlamings-Wagenaars

Elisabeth-TweeSteden Ziekenhuis

Anne van Oudheusden

Elisabeth-TweeSteden Ziekenhuis

Pascal De Waegemaeker

Universitair Ziekenhuis Gent

Isabel Leroux-Roels

Universitair Ziekenhuis Gent

Martine Verelst

Katholieke Universiteit Leuven Universitaire Ziekenhuizen Leuven

Evelien Maas

ZorgSaam

Anita van Oosten

Admiraal De Ruyter Ziekenhuis

Patricia Willemse

Elkerliek Ziekenhuis

Esther van Asselen

Elkerliek Ziekenhuis

Ella Klomp-Berens

Maastricht Universitair Medisch Centrum+

\section{Karen Franssen}

Maastricht Universitair Medisch Centrum+

Elise Van Cauwenberg

Universitair Ziekenhuis Antwerpen

Jan Kluytmans 


\section{Research}

Keywords: ATP measurement, fomite, surface contamination, cleaning

Posted Date: December 16th, 2019

DOI: https://doi.org/10.21203/rs.2.18947/v1

License: (9) This work is licensed under a Creative Commons Attribution 4.0 International License. Read Full License

Version of Record: A version of this preprint was published at Antimicrobial Resistance and Infection Control on May 28th, 2020. See the published version at https://doi.org/10.1186/s13756-020-00730-9. 


\section{Abstract}

Background The objective of this study was to determine the level of environmental contamination in hospitals in the Dutch/Belgian border area, using ATP measurements.

Design A cross-sectional observational survey Methods Standardized ATP measurements were conducted in 9 hospitals on 32 hospital wards. Thirty pre-defined surfaces per hospital ward were measured with the $3 \mathrm{M}$ Clean Trace NG luminometer. Results are displayed in relative light units (RLU). RLU > 1000 was considered as "not clean." Differences in RLU values were compared between countries, hospitals, fomite groups and medical specialties.

Results A total of 960 ATP measurements were performed, ranging from 60 up to 120 per hospital. The median RLU-value was 568 (range: $3-277,586$ ) and $37.7 \%$ of the measurements were rated as not clean (RLU >1000). There were significant differences between countries, hospitals and fomite groups.

Conclusion ATP measurements can be used as a more objective approach to determine the level of environmental contamination in hospitals. Significant differences in ATP levels were found between hospitals and between countries. Also, substantial differences were found between different fomite groups. These findings offer potential targets for improvement of cleanliness in healthcare facilities.

\section{Background}

Contaminated surfaces and fomites are considered and important reservoir of (multi-resistant) microorganisms in hospitals. ${ }^{1,2,3}$ Therefore, cleaning of the environment is important for reducing bacterial spread, controlling antimicrobial resistance and improving patient safety.

The assessment of the cleanliness of surfaces in hospitals is mostly conducted by visual inspection. This method is not sensitive and subjective and therefore unreliable. ${ }^{4,5,6}$ Recently, a more objective technique was introduced to measure biological contamination. This technique is based on the measurement of adenosine triphosphate (ATP), a molecule that is present in all organic cells. The amount of ATP measured is expressed in relative light units (RLU) using the 3M Clean Trace NG luminometer: the higher the amount of ATP measured, the higher the RLU value will be. ATP measurement seems a promising alternative to visual inspection and aerobic colony count cultures. ${ }^{7}$

The aim of this study was to determine the level of environmental contamination in hospitals in the Dutch/Belgian border area as a part of the cross border One Health project that aimed to control the spread of antimicrobial resistance (the i-4-1-Health project). Differences between countries, hospitals, fomite groups and medical specialties were investigated and visualized.

\section{Methods}

\section{Setting}


As part of a multicenter One Health project in the Dutch/Belgian border area, the i-4-1-Health project, standardized ATP measurements were conducted in 9 hospitals (3 Belgian university hospitals, 1 Dutch university hospital, 3 Dutch teaching hospitals and 2 Dutch general hospitals). The ATP measurements were conducted on different hospital wards, from 2 up to 4 wards per hospital, depending on the hospital size. In each hospital, ATP measurements were conducted on at least a surgical ward and an internal medicine ward. When ATP measurements were conducted on more than 2 wards a selection was made from the medical specialties urology, cardiology, orthopedic surgery, pulmonology and/or geriatrics. On each ward, ATP measurements were performed on 30 pre-defined fomites (table 1). These fomites were classified into 4 different groups: medical devices, patient bound materials, sanitary items and ward bound materials. Fomites were chosen based on the following criteria: frequently touched by nursing staff or frequently touched by patients or in the direct vicinity of patients or high-risk surfaces (e.g. tabletop for medication preparation).

\section{Table 1:}

Overview of the fomites measured per hospital ward. 


\begin{tabular}{|l|l|}
\hline Fomite & \multicolumn{1}{|c|}{ Medical devices } \\
\hline Blood pressure meter - control panel & \\
\hline Thermometer & \\
\hline Glucose meter - control panel & \\
\hline Glucose meter - insertion opening & \\
\hline Infusion stand x3 & \multirow{3}{*}{ Patient bound materials } \\
\hline Stethoscope - membrane & \\
\hline Infusion pump - control panel x2 & \\
\hline Pull-up bracket & \\
\hline Nightstand - pullout tabletop & \\
\hline Bedrails & \\
\hline Paging system at bed x2 & \\
\hline Toilet - seat & \\
\hline Toilet - bowl & \\
\hline Toilet - flush button & \\
\hline Toilet - support/bracket & \\
\hline Toilet chair - seat & \\
\hline Bedpan cleaner - control panel & \\
\hline Sink - faucet operation x2 & \\
\hline Shower - support/bracket & \\
\hline Shower - showerhead & \\
\hline Keyboard - Computer On Wheels (COW) & \\
\hline Keyboard - team post & \\
\hline Tabletop medication preparation & \\
\hline Telephone - keys & \\
\hline Chair - seat & \\
\hline
\end{tabular}

\section{ATP measurements}

The Clean-Trace NG Luminometer (3M, Zoeterwoude, the Netherlands) was used for the ATP measurements, results were reported in RLU. ATP measurements were conducted by trained researchers working at the department of infection control of the corresponding hospital. Two methods of measurement were performed. Method A: a surface of approximately $100 \mathrm{~cm}^{2}(10 \times 10 \mathrm{~cm})$ is thoroughly swabbed in two directions with an ATP-swab. Method B: the whole surface is thoroughly swabbed with an ATP-swab, and the $100 \mathrm{~cm}^{2}$ surface is approached as best as possible. Method B was used for fomites that did not have a flat surface, an easily measureable surface or a surface smaller than 100 $\mathrm{cm}^{2}$. The manufacturer's instructions on conducting the ATP measurements were followed. Each researcher got instructed to perform the ATP measurements around noon. Instruction was given to not perform ATP measurement directly after cleaning. 


\section{RLU breakpoints}

Within the i-4-1-Health project RLU breakpoints were defined by a group of experts based on the literature and previous experience with ATP measurements in hospitals. ${ }^{7}$ These breakpoints were used to facilitate feedback to the wards in a way that would be better understood. The following breakpoints were chosen: clean (RLU $<1000)$, intermediate ( $R L U \geq 1000$ to $<3000)$, dirty $(R L U \geq 3000$ to $<10,000)$ and extremely dirty $(R L U \geq 10,000)$. For these breakpoints color codes were used to visualize the level of contamination (respectively green, orange, red and black).

\section{Statistical methods}

All data were analyzed with Statistical Package for Social Science software (SPSS; IBM Corp., Armonk, New York, US; version 25). Differences in the distribution of RLU values between hospitals and fomite groups were calculated using the Kruskall Wallis test, adjustment for multiple testing was performed. Overall difference between both countries and medical specialties was calculated using the Mann-Whitney U test. Because of the large differences in number of measurements per medical specialty, two groups were formed: surgical and non-surgical specialties. Statistical significance was accepted at $\mathrm{p}<0.05$ after correction for multiple testing. Relative Risks (RRs) for the more frequent occurrence of "not clean" fomites (RLU >1000) were calculated with univariable and multivariable generalized linear models (GLM) with a binomial distribution.

\section{Results}

In total 960 ATP measurements were performed, 30 ATP measurements per ward, accounting for 60 up to 120 ATP measurements per hospital. The median RLU-value was 568 with a range from 3 up to 277,586.

Of all measurements 37.7\% (362/960) were considered as "not clean" (RLU >1000), 12.7\% (122/960) had RLU values above 3000 ('dirty') and 3.8\% (37/960) above 10,000 ('extremely dirty').

Figure 1 shows the differences in median RLU-values between the 9 hospitals in both countries. The pvalues of the pairwise comparison of hospitals are visualized in table 2. Significant differences are highlighted. The median RLU-value per hospital from high to low was: $2137,1131,872,835,807,524$, 
455, 294, 278. Hospital 1 had significantly lower ATP levels than all other hospitals apart from hospital 2 and 3. On the other hand, hospital 9 had significantly higher values than all other hospitals. The median RLU-value measured in the Netherlands was 793, the median RLU-value measured in Belgium was 431. The difference in RLU distribution between the two countries was significant $(p<0.001)$.

Table 2:

Matrix of p-values of pairwise comparisons between different hospitals.

\begin{tabular}{l|c|c|c|c|c|c|c|c|c|}
\cline { 2 - 9 } & $\begin{array}{c}\text { Hospital } \\
9\end{array}$ & $\begin{array}{c}\text { Hospital } \\
8\end{array}$ & $\begin{array}{c}\text { Hospital } \\
7\end{array}$ & $\begin{array}{c}\text { Hospital } \\
6\end{array}$ & $\begin{array}{c}\text { Hospital } \\
5\end{array}$ & $\begin{array}{c}\text { Hospital } \\
4\end{array}$ & $\begin{array}{c}\text { Hospital } \\
3\end{array}$ & $\begin{array}{c}\text { Hospital } \\
2\end{array}$ & $\begin{array}{c}\text { Hospital } \\
1\end{array}$ \\
\hline spital & & $0.029^{*}$ & $0.010^{*}$ & $0.020^{*}$ & $0.013^{*}$ & $0.000^{*}$ & $0.000^{*}$ & $0.000^{*}$ & $0.000^{*}$ \\
\hline spital & $0.029^{*}$ & & 1.000 & 1.000 & 1.000 & 1.000 & 0.082 & $0.000^{*}$ & $0.000^{*}$ \\
\hline spital & $0.010^{*}$ & 1.000 & & 1.000 & 1.000 & 1.000 & 0.254 & $0.000^{*}$ & $0.000^{*}$ \\
\hline spital & $0.020^{*}$ & 1.000 & 1.000 & & 1.000 & 1.000 & 1.000 & $0.002^{*}$ & $0.001^{*}$ \\
\hline spital & $0.013^{*}$ & 1.000 & 1.000 & 1.000 & & 1.000 & 1.000 & $0.000^{*}$ & $0.000^{*}$ \\
\hline spital & $0.000^{*}$ & 1.000 & 1.000 & 1.000 & 1.000 & & 1.000 & $0.007^{*}$ & $0.002^{*}$ \\
\hline spital & $0.000^{*}$ & 0.082 & 0.254 & 1.000 & 1.000 & 1.000 & & 0.263 & 0.093 \\
\hline spital & $0.000^{*}$ & $0.000^{*}$ & $0.000^{*}$ & $0.002^{*}$ & $0.000^{*}$ & $0.007^{*}$ & 0.263 & & 1.000 \\
\hline spital & $0.000^{*}$ & $0.000^{*}$ & $0.000^{*}$ & $0.001^{*}$ & $0.000^{*}$ & $0.002^{*}$ & 0.093 & 1.000 & \\
\hline
\end{tabular}

\section{* indicates a statistically significant difference $(p<0.05)$.}

Per fomite group 160 to 320 ATP measurements were conducted: 320 ATP measurements in the medical devices group, 320 ATP measurements in the sanitary items group, 160 ATP measurements in the patient bound materials group and 160 ATP measurements in the ward bound materials group.

The differences in median RLU-value between the different fomite groups are visualized in figure 2 . The pairwise comparisons of the fomite groups are visualized in table 3, significant differences are highlighted. The median RLU-value was 931 in the patient bound materials group, 659 in ward bound 
materials, 651 in medical devices, and 396 in sanitary items. Sanitary items had significantly lower values than all other groups of fomites.

\section{Table 3:}

Matrix of p-values of pairwise comparisons between different categories of fomites.

\begin{tabular}{l|c|c|c|c|}
\cline { 2 - 5 } & Patient bound materials & Medical devices & Ward bound materials & Sanitary items \\
\hline ient bound materials & & 0.087 & 0.487 & $0.000^{*}$ \\
\hline dical devices & 0.087 & & 1.000 & $0.006^{*}$ \\
\hline rd bound materials & 0.487 & 1.000 & & $0.011^{*}$ \\
\hline itary items & $0.000^{*}$ & $0.006^{*}$ & $0.011^{*}$ & \\
\hline
\end{tabular}

* indicates a statistically significant difference $(p<0.05)$.

Per medical specialty 30 to 270 ATP measurements were conducted. The surgical group consisted out of 450 measurements, the non-surgical group out of 510 measurements. The median RLU-value measured in the surgical group was 626 , the median RLU-value measured in the non-surgical groups was 545 . The difference in RLU distribution between the two was not significant ( $p>0.05)$.

Univariate predictors for the more frequent occurrence of "not clean" surfaces are visualized in table 4, significant differences are highlighted.

Multivariate predictors for a higher chance of a non-clean surface were hospital 3 until 9 and all fomite groups with sanitary items as reference.

\section{Table 4:}

Univariate and multivariate analysis per group with display of percentages of "not clean" (RLU >1000) items. 


\begin{tabular}{llllll}
\hline & & Univariate & & Multivariate \\
& $>1000 \mathrm{RLU}(\%)$ & $P$ & $\mathrm{RR}(95 \% \mathrm{BI})$ & $P$ & $\operatorname{RR}(95 \% \mathrm{BI})$ \\
\hline
\end{tabular}

\begin{tabular}{lccccc}
\hline $\begin{array}{l}\text { hospital } \\
\text { hospital 1 }\end{array}$ & 15.8 & Ref & & \\
\hline hospital 2 & 19.2 & 0.498 & $1.21(0.70-2.10)$ & 0.473 & $1.25(0.70-2.13)$ \\
\hline hospital 3 & 30.0 & 0.011 & $\mathbf{1 . 8 9}(1.15-3.11)$ & 0.011 & $1.90(1.16-3.11)$ \\
\hline hospital 4 & 35.8 & 0.001 & $2.26(1.40-3.65)$ & 0.001 & $2.28(1.38-3.58)$ \\
\hline hospital 5 & 47.5 & $\mathbf{0 . 0 0 0}$ & $\mathbf{3 . 0 0}(1.91-4.72)$ & 0.000 & $2.95(1.88-4.64)$ \\
\hline hospital 6 & 40.0 & $\mathbf{0 . 0 0 0}$ & $\mathbf{2 . 5 3 ( 1 . 5 1 - 4 . 2 3 )}$ & 0.001 & $3.37(2.14-5.21)$ \\
\hline hospital 7 & 45.0 & $\mathbf{0 . 0 0 0}$ & $\mathbf{2 . 8 4 ( 1 . 8 0 - 4 . 4 9 )}$ & 0.000 & $2.44(1.45-4.08)$ \\
\hline hospital 8 & 52.5 & $\mathbf{0 . 0 0 0}$ & $\mathbf{3 . 3 2}(2.12-5.18)$ & 0.000 & $2.78(1.76-4.39)$ \\
\hline hospital 9 & 71.7 & $\mathbf{0 . 0 0 0}$ & $\mathbf{4 . 5 3 ( 2 . 9 1 - 7 . 0 4 )}$ & 0.000 & $4.38(2.82-6.80)$ \\
\hline
\end{tabular}

\begin{tabular}{lccccc}
$\begin{array}{l}\text { medical specialty } \\
\text { surgical }\end{array}$ & 40.7 & Ref & & & \\
\hline non-surgical & 35.1 & 0.076 & $0.86(0.73-1.01)$ & 0.241 & $0.91(0.78-1.06)$
\end{tabular}

\begin{tabular}{|c|c|c|c|c|c|}
\hline $\begin{array}{l}\text { surface category } \\
\text { sanitary items }\end{array}$ & 29.4 & Ref & & & \\
\hline Patient bound materials & 47.5 & 0.000 & $1.62(1.28-2.05)$ & 0.000 & $1.57(1.27-1.93)$ \\
\hline ward bound & 40.6 & 0.010 & $1.39(1.08-1.78)$ & 0.008 & $1.37(1.09-1.72)$ \\
\hline \multicolumn{6}{|l|}{ materials } \\
\hline medical devices & 38.4 & 0.016 & $1.30(1.05-1.62)$ & 0.014 & $1.30(1.05-1.60)$ \\
\hline
\end{tabular}

Significant differences in bold $(p<0.05)$.

\section{Conclusion}

Within this study significant differences in environmental contamination were found between countries, hospitals and fomite groups. In addition a high percentage of "not clean" (>1000 RLU) surfaces or fomites was found.

In all hospitals there is room for improvement, but this varies considerably between hospitals. After adjusting for medical specialty and fomite group the relative risk of the hospital with the highest proportion of fomites which were "not clean" was 4.4 times higher than the hospital with the lowest proportion. We found a high level of variation of "not clean" surfaces between groups (e.g. hospitals, fomite groups). These results can be used to improve cleanliness by defining best practices and implementing them. For instance, by analyzing cleaning regimes (cleaning method, cleaning staff, products used for cleaning and disinfection, standard disinfection during hospital stay and/or after discharge, etc.) in the hospitals with a lower level of environmental contamination can help to improve cleaning regimes in hospitals with higher levels of environmental contamination. Also, by analyzing different fomites and fomite groups, cleaning can be improved by focusing on the most contaminated fomites. 


\section{Discussion}

Hospital cleanliness is an important factor to reduce bacterial spread and therefore prevent hospital infections. ${ }^{1,2,3}$ Measuring hospital cleanliness can be time consuming and judging surface contamination by visual assessment alone is an un reliable indicator of the level of environmental contamination. ${ }^{4,5,6}$ ATP measurements seem a promising alternative to visual assessments by quantifying the amount of organic tissue on a surface in objective and reproducible way. The results are available practically on the spot and with the cut offs that we defined before the project started the RLU's are easy to understand for the users. ${ }^{8}$

Nevertheless, there is still an ongoing discussion if ATP measurements are suitable to quantify the level of bacterial contamination of a surface. This because ATP reflects the amount of all organic material and not only bacteria. ${ }^{9}$ Critics argue that a relatively low level of ATP solely based on the presence of bacteria only, may carry a relatively high risk to patients. This is without doubt a valid argument. On the other hand the amount of organic material does reflect the level of environmental contamination and therefore can be used as a surrogate marker to measure the effectiveness of cleaning. In addition, organic material may serve as a nutritional source for bacteria and thereby promote bacterial growth. As an example, vancomycin resistant enterococci (VRE) are frequently found on inanimate surfaces which are shown to be a reservoir and a cause of spread of VRE in the hospital environment. ${ }^{10,11}$ Thus by identifying dirty surfaces with ATP measurements, it may be possible to reduce spread of VRE or other multi resistant bacteria. ${ }^{1}$ We consider the ATP measurement as a useful tool to measure the level of environmental contamination in a reliable and reproducible way. Thereby they can be used to benchmark hospitals or wards and improve the cleanliness of hospitals.

We defined ATP thresholds, based on literature review and on previous ATP measurements in the participating hospitals before the project started. ${ }^{12}$ Other studies have recommended an RLU threshold for cleanliness at 250-500 RLU, however this threshold is intended for measurement (almost) directly after cleaning. 4,6,8,13,14,15 We developed ATP thresholds for conducting an ATP measurement at a random point in time on a hospital ward, not knowing if items were used or cleaned that day. The goal of this study was to visualize the environmental contamination independent from the time of cleaning and to determine the environmental contamination to which a patient is exposed in the hospital. The hospital cleaning protocols were not monitored as part of this study. The main goal was to use the defined RLU breakpoints for insight in surface contamination for improvement of cleaning in a later phase of the project. A group of experts (experienced infection control practitioners and microbiologists) defined the thresholds.

There are some (potential) limitations of this study. First, different researchers measured fomites at different points in time. This can cause bias because a researcher could have his/her own method of sampling and for instance choose spots which look visually cleaner or dirtier. However, researchers were given a training and instructions on how to perform the measurements properly, according to manufacturer's guidelines. Also, the researchers checked and validated each other before the project was 
started. Instructions were given on how to swab each fomite. Also, researchers were given instructions to perform the ATP measurements early in the afternoon to standardize the timing.

The main advantages of ATP measurements are the objective and reproducible results, which are produced on the spot so provide immediate feedback. ATP measurements give a quantitative result, which is easy to interpret by nursing or cleaning staff when thresholds for clean and not clean are defined. In comparison, aerobic colony counts give an indication of the number of viable bacteria. This may be considered more relevant but the major disadvantage is that the results take $24-48 \mathrm{~h}$ to become available to those who can improve the cleaning process. This makes it less attractive for quality improvement using rapid feedback to the users.

ATP measurements can be used as a fast and objective approach to determine the level of environmental contamination in hospitals. The substantial and significant differences between countries, hospitals and fomite groups provide a basis for improvement. Further research in cleaning regimes is needed to explain the differences between the hospitals. Subsequent changes in the cleaning policy can be judged for their effectiveness using repeated ATP measurements.

\section{Declarations}

\section{Ethics approval and consent to participate}

Not applicable.

\section{Consent for publication}

Not applicable.

\section{Availability of data and materials}

As agreed within the i-4-1-Health consortium, the i-4-1-Health datasets will be made available no earlier than December 31st, 2020 and no later than December 31st, 2024, in accordance with the FAIR (Findable, Accessible, Interoperable and Reusable) data principles. ${ }^{16}$

\section{Competing interests}

The authors declare that they have no competing interests. 


\section{Funding}

The i-4-1-Health project was financed by the Interreg V Flanders-The Netherlands program, the crossborder cooperation program with financial support from the European Regional Development Fund (ERDF). Additional financial support was received from the Dutch Ministry of Health, Welfare and Sport, the Dutch Ministry of Economic Affairs, the Province of Noord-Brabant, the Belgian Department of Agriculture and Fisheries, the Province of Antwerp and the Province of East-Flanders. ATP luminometers and ATP surface swabs were provided by $3 \mathrm{M}$. The authors are free to publish the results from the project without interference from the funding bodies or $3 \mathrm{M}$.

\section{Authors' contributions}

Andreas van Arkel was responsible for data analysis and writing of the manuscript. The manuscript was judged by Ina Willemsen, Jan Kluytmans, Pascal De Waegemaeker, Isabel Leroux-Roels and Martine Verelst. Sindy Vlamings-Wagenaars, Anne van Oudheusden, Pascal De Waegemaeker, Martine Verelst, Evelien Maas, Anita van Oosten, Patricia Willemse, Esther van Asselen, Ella Klomp-Berens, Karen Franssen and Elise Van Cauwenberg were responsible for collection of the data. All authors read and approved the final manuscript.

\section{Acknowledgements}

We are grateful to the infection control practitioners in the participating hospitals for their contribution to the collection of the epidemiological data.

\section{i-4-1-Health Study Group}

Lieke van Alphen (Maastricht University Medical Center+, Maastricht, the Netherlands), Nicole van den Braak (Avans University of Applied Sciences, Breda, the Netherlands), Caroline Broucke (Agency for Care and Health, Brussels, Belgium), Anton Buiting (Elisabeth-TweeSteden Hospital, Tilburg, the Netherlands), Liselotte Coorevits (Ghent University Hospital, Ghent, Belgium), Sara Dequeker (Agency for Care and Health, Brussels, Belgium and Sciensano, Brussels, Belgium), Jeroen Dewulf (Ghent University, Ghent, Belgium), Wouter Dhaeze (Agency for Care and Health, Brussels, Belgium), Bram Diederen (ZorgSaam Hospital, Terneuzen, the Netherlands), Helen Ewalts (Regional Public Health Service Hart voor Brabant, Tilburg, the Netherlands), Herman Goossens (University of Antwerp, Antwerpen, Belgium and Antwerp University Hospital, Antwerp, Belgium), Inge Gyssens (Hasselt University, Hasselt, Belgium), Casper den Heijer (Regional Public Health Service Zuid-Limburg, Heerlen, the Netherlands), Christian Hoebe (Maastricht University Medical Center+, Maastricht, the Netherlands and Regional Public Health Service 
Zuid-Limburg, Heerlen, the Netherlands), Casper Jamin (Maastricht University Medical Center+, Maastricht, the Netherlands), Patricia Jansingh (Regional Public Health Service Limburg Noord, Venlo, the Netherlands), Jan Kluytmans (Amphia Hospital, Breda, the Netherlands and University Medical Center Utrecht, Utrecht University, Utrecht, the Netherlands), Marjolein Kluytmans-van den Bergh (Amphia Hospital, Breda, the Netherlands and University Medical Center Utrecht, Utrecht University, Utrecht, the Netherlands), Stefanie van Koeveringe (Antwerp University Hospital, Antwerp, Belgium), Sien De Koster (University of Antwerp, Antwerp, Belgium), Christine Lammens (University of Antwerp, Antwerp, Belgium), Isabel Leroux-Roels (Ghent University Hospital, Ghent, Belgium), Hanna Masson (Agency for Care and Health, Brussel, Belgium), Ellen Nieuwkoop (Elisabeth-TweeSteden Hospital, Tilburg, the Netherlands), Anita van Oosten (Admiraal De Ruyter Hospital, Goes, the Netherlands), Natascha Perales Selva (Antwerp University Hospital, Antwerp, Belgium), Merel Postma (Ghent University, Ghent, Belgium), Stijn Raven (Regional Public Health Service West-Brabant, Breda, the Netherlands), Veroniek Saegeman (University Hospitals Leuven, Leuven, Belgium), Paul Savelkoul (Maastricht University Medical Center+, Maastricht, the Netherlands), Annette Schuermans (University Hospitals Leuven, Leuven, Belgium), Nathalie Sleeckx (Experimental Poultry Centre, Geel, Belgium), Krista van der Slikke (Regional Public Health Service Zeeland, Goes, the Netherlands), Arjan Stegeman (Utrecht University, Utrecht, the Netherlands), Tijs Tobias (Utrecht University, Utrecht, the Netherlands), Paulien Tolsma (Regional Public Health Service Brabant Zuid-Oost, Eindhoven, the Netherlands), Jacobien Veenemans (Admiraal De Ruyter Hospital, Goes, the Netherlands), Dewi van der Vegt (PAMM Laboratory for Pathology and Medical Microbiology, Veldhoven, the Netherlands), Martine Verelst (University Hospitals Leuven, Leuven, Belgium), Carlo Verhulst (Amphia Hospital, Breda, the Netherlands), Pascal De Waegemaeker (Ghent University Hospital, Ghent, Belgium), Veronica Weterings (Amphia Hospital, Breda, the Netherlands), Clementine Wijkmans (Regional Public Health Service Hart voor Brabant, Tilburg, the Netherlands), Patricia Willemse-Smits (Elkerliek Hospital, Helmond, the Netherlands), Ina Willemsen (Amphia Hospital, Breda, the Netherlands.)

\section{References}

1. Hayden MK, Bonten MJ, Blom DW, et al. Reduction in acquisition of vancomycin-resistant enterococcus after enforcement of routine environmental cleaning measures. Clin Infect Dis. 2006;42:1552-60.

2. Dancer SJ. Importance of the Environment in Methicillin-resistant Staphylococcus aureus Acquisition: the Case for Hospital Cleaning. Lancet Infect Dis. 2008;8:101-13.

3. Boyce JM. Environmental contamination makes an important contribution to hospital infection. J. Hosp Infect. 2007;65 Suppl 2:50-4.

4. Griffith CJ, Obee P, Cooper RA, et al. The effectiveness of existing and modified cleaning regimens in a Welsh hospital. J. Hosp Infect. 2007;66:352-9.

5. Cooper RA, Griffith CJ, Malik RE, et al. Monitoring the effectiveness of cleaning in four British hospitals. Am J Infect Control. 2007;35:33841. 
6. Griffith CJ, Cooper RA, Gilmore J, et al. An evaluation of hospital cleaning regimes and standards. J. Hosp Infect. 2000;45:19-28.

7. Sherlock $\mathrm{O}, \mathrm{O}$ 'Connell N, Creamer E, et al. Is it really clean? An evaluation of the efficacy of four methods for determining hospital cleanliness. J Hosp Infect. 2009;doi:10.1016/j.jhin.2009.02.013.

8. Lewis T, Griffith $C$, Gallo M, et al. A modified ATP benchmark for evaluating the cleaning of some hospital environmental surfaces. J. Hosp Infect. 2008;doi:10.1016/j.jhin.2008.03.013.

9. Willis $\mathrm{C}$, Morley R, Westbury $\mathrm{J}$, et al. Evaluation of ATP bioluminescence swabbing as a monitoring and training tool for effective hospital cleaning. Journal of Infection Prevention. 2007;8:17-21.

10. Lee AS, White E, Monahan LG, et al. Defining the Role of the Environment in the Emergence and Persistence of vanA Vancomycin-Resistant Enterococcus (VRE) in an Intensive Care Unit: A Molecular Epidemiological Study. Infect Control Hosp Epidemiol. 2018; doi:10.1017/ice.2018.29.

11. Porwancher R, Sheth A, Remphrey S, et al. Epidemiological study of hospital-acquired infection with vancomycin-resistant Enterococcus faecium: possible transmission by an electronic ear-probe thermometer. Infect Control Hosp Epidemiol. 1997;18:771-3.

12. Willemsen I, Kluytmans J. The infection risk scan (IRIS): standardization and transparency in infection control and antimicrobial use. Antimicrob Resist Infect Control. 2018; doi:10.1186/s13756018-0319-z.

13. Amin SR, Folkert CM, Erie JC. Assessing the effectiveness of surface cleaning methods in intravitreal injection procedure rooms. Ophthalmology. 2014;doi:10.1016/j.ophtha.2013.08.006.

14. Boyce JM, Havill NL, Mangione E, et al. Comparison of fluorescent marker systems with 2 quantitative methods of assessing terminal cleaning practices. Infect Control Hosp Epidemiol. 2011; doi:10.1086/662626.

15. Moore G, Smyth D, Singleton J, et al. The use of adenosine triphosphate bioluminescence to assess the efficacy of a modified cleaning program implemented within an intensive care setting. Am J Infect Control. 2010; doi:10.1016/j.ajic.2010.02.011.

16. Wilkinson MD, Dumontier $M$, Aalbersberg IJ, et al. The FAIR guiding principles for scientific data management and stewardship. Sci Data 2016; doi:10.1038/sdata.2016.18.

\section{Figures}




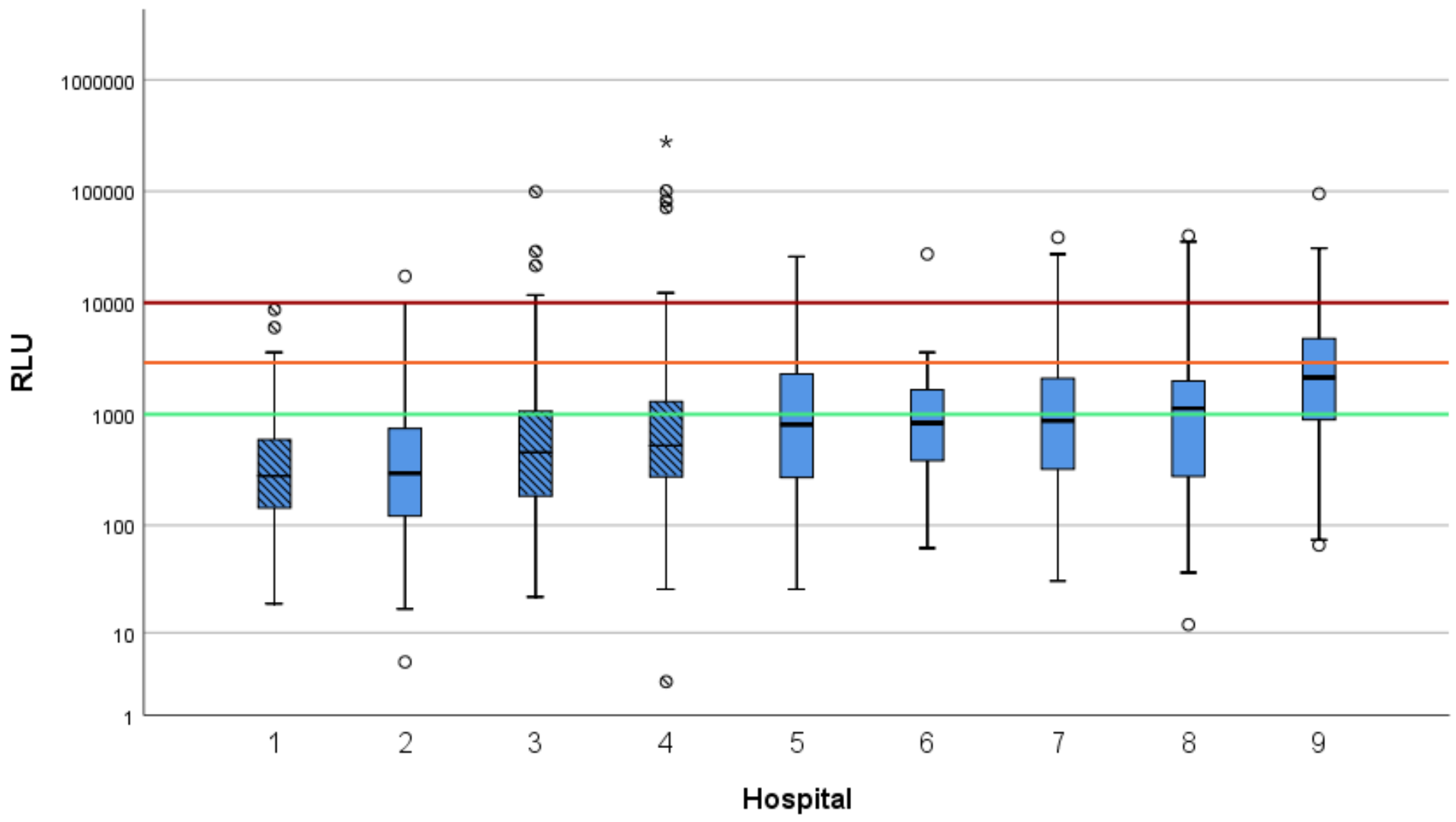

\section{Figure 1}

Boxplot of RLU values between hospitals with RLU breakpoints. Legend: Belgian hospitals are shaded. Outliers are marked with a circle, extreme outliers with a star. RLU breakpoints are marked by colored lines. 


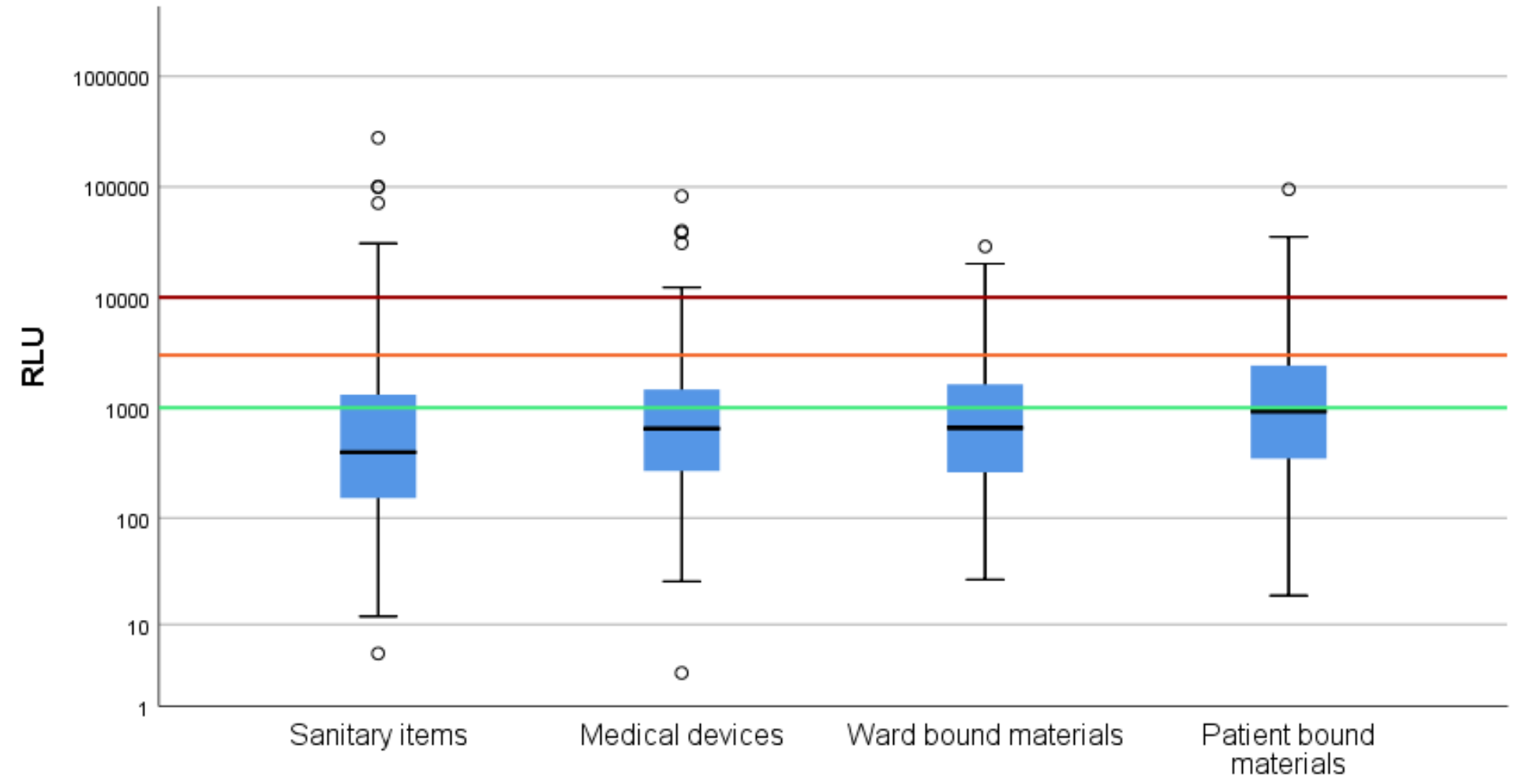

Figure 2

Boxplot of RLU values between fomite groups with RLU breakpoints. Legend: Outliers are marked with a circle. RLU breakpoints are marked by colored lines. 